\title{
Extreme Elevation of Alkaline Phosphatase in a Pregnancy Complicated by Uncontrolled Chronic Hypertension and Its Association With Placental Pathology
}

\author{
Ena Arora ${ }^{\mathrm{a}, \mathrm{c}}$, Tracey Juron ${ }^{\mathrm{a}}$, Aleksandr Fuks ${ }^{\mathrm{a}}$, Carolyn Salafia ${ }^{\mathrm{b}}$
}

\begin{abstract}
Alkaline phosphatase (ALP) is an enzyme produced primarily by cells of liver, bone, kidney, small intestine and placenta, each detectable by a specific isoenzyme. It appears in maternal serum in the second trimester and increases progressively with gestational age. Review of the literature has linked markedly elevated serum ALP levels with adverse obstetric and perinatal outcomes, including preterm labor, hypertensive disorders of pregnancy and low birth weight. The exact mechanism of this association is unknown, but an overwhelming majority of literature suggests placental dysfunction as the primary cause. We describe a 38-year-old G5P2022 female who began prenatal care at $10+0 / 7$. She started on labetalol $100 \mathrm{mg}$ BID at $17+0 / 7$. At $35+5 / 7$, she had persistent severely elevated blood pressure, with ALP of 2,826 U/L. She was admitted for observation, and labetalol titrated to $300 \mathrm{mg}$ BID. The maximum value of ALP was $3,429 \mathrm{U} / \mathrm{L}$ at $36+5 / 7$. Serum isoenzyme assay revealed solely placental origin. Labor induction was done at $37+0 / 7$ followed by delivery of a 3,315 g neonate, with a displaced clavicular fracture in the absence of shoulder dystocia. Placental pathology revealed diffuse villous edema and chorionic plate vessel with dense embedded mural thrombus. Our case represents an association between extreme elevation of ALP in pregnancy and placental dysfunction, confirmed by placental pathology. Close monitoring of the absolute value and degree of rise in ALP may hold significant clinical importance in predicting impaired placental function and ultimately in determining the timing of delivery.
\end{abstract}

Keywords: Alkaline phosphatase; Hypertension; Placental dysfunction; Pathology

Manuscript submitted January 6, 2022, accepted January 19, 2022

Published online February 15, 2022

aDepartment of OB and GYN, Icahn School of Medicine at Mount Sinai (NYC Health + Hospitals/Queens), New York City, NY, USA

${ }^{b}$ Department of Pathology, Icahn School of Medicine at Mount Sinai (NYC Health + Hospitals/Queens), New York City, NY, USA

${ }^{\mathrm{c} C}$ Corresponding Author: Ena Arora, Department of OB and GYN, Icahn School of Medicine at Mount Sinai (NYC Health + Hospitals/Queens), New York City, NY 11432, USA. Email: Aroraena@gmail.com

doi: https://doi.org/10.14740/jem789
Introduction

Alkaline phosphatase (ALP) is a plasma membrane-bound glycoprotein involved in energy production. Human ALP is classified into tissue-specific forms or isozymes, in accordance with the site where it is expressed: placenta, intestine, liver, bone, kidney and germ cells [1]. During pregnancy, serum ALP levels increase progressively with a maximum value reached in the third trimester [2]. Similarly, heat-stable placental alkaline phosphatase (PALP) isoenzyme increases gradually throughout pregnancy, reaching a peak level in the predelivery period (164 - $495 \mathrm{U} / \mathrm{L})$, contributing to an increase in the total alkaline phosphatase (ALPt) activity. ALP isoenzyme activity decreases to the normal level at about 20 weeks postpartum. In pregnancy, significantly elevated levels of serum ALP require careful investigation. Characterization by electrophoresis should be performed to determine the origin of ALP. Several studies suggest an association between elevated ALP activity and preeclampsia [3-6], low birth weight, prematurity and placental insufficiency [7-9]. We present a case report of a 38-year-old female with uncontrolled chronic hypertension and extreme elevation of ALP and its association with placental dysfunction, demonstrated by placental pathology.

\section{Case Report}

\section{Investigations}

A 38-year-old G5P2022 female diagnosed with chronic hypertension in 2018 presented to us for prenatal care at 10 weeks gestation. On initial presentation to our institution, she was not taking antihypertensives. Vitally, she was hypertensive with blood pressure (BP) of 166/94 mm Hg. She was asymptomatic and clinical examination was unremarkable. Baseline hepatic function panel and ALP were within normal limits (ALP 75 $\mathrm{U} / \mathrm{L}$ ), as well as a $24-\mathrm{h}$ urine protein of $126 \mathrm{mg}$ and a normal 2D echocardiogram. She began prophylactic aspirin $81 \mathrm{mg}$ daily at 12 weeks and was placed on labetalol $100 \mathrm{mg}$ BID at 17 weeks for BP control. Her prenatal course remained unremarkable until 36 weeks, when she was noted to have persistent severely elevated BP readings with systolic BP $>160$ $\mathrm{mm} \mathrm{Hg}$. 


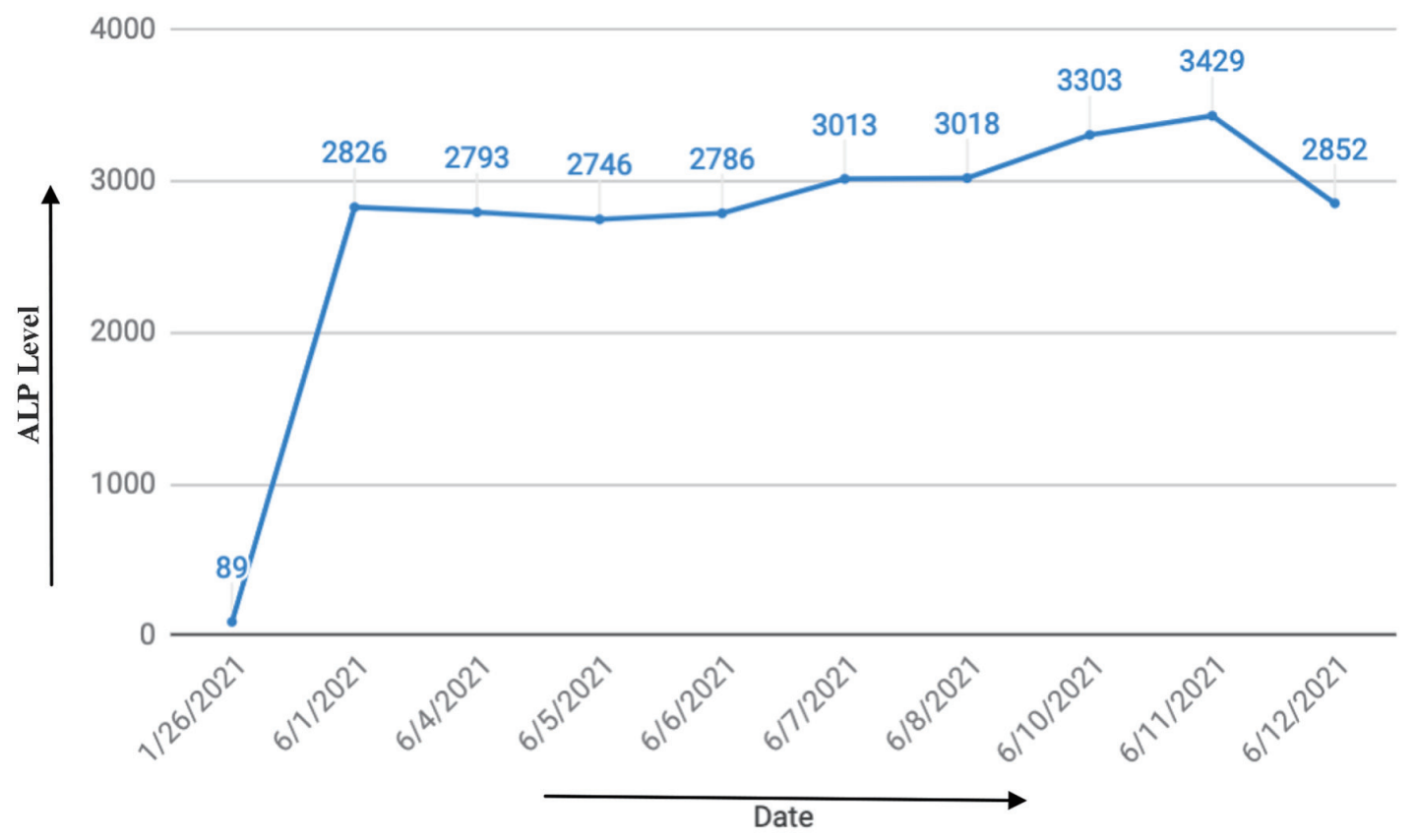

Figure 1. Trend of alkaline phosphatase (ALP) over time.

\section{Diagnosis}

A 38-year-old G5P2022 female with uncontrolled chronic hypertension with severely elevated ALP levels at 36 weeks gestation was admitted for BP control.

\section{Treatment}

She was admitted for observation with up-titration of labetalol to $300 \mathrm{mg}$ BID and the addition of nifedipine XL 30 daily. On admission, her ALP level was noted to be markedly elevated at 2,826 U/L. Serial measurements of ALP were performed. Peak value of 3,429 U/L was noted at 37 weeks (Fig. 1). Transami- nases remained within normal limits. Right upper quadrant sonogram was normal. Serum ALP isoenzyme assay revealed solely placental origin. She underwent induction of labor at 37 weeks +0 day with magnesium sulfate for seizure prophylaxis intrapartum and for $24 \mathrm{~h}$ postpartum.

\section{Follow-up and outcomes}

She delivered a 3,315 g neonate with a displaced clavicular fracture, in the absence of shoulder dystocia. Placental pathology revealed diffuse villous edema and chorionic plate vessels with dense embedded mural thrombus (Fig. 2). Our case represents an association between extreme elevation of
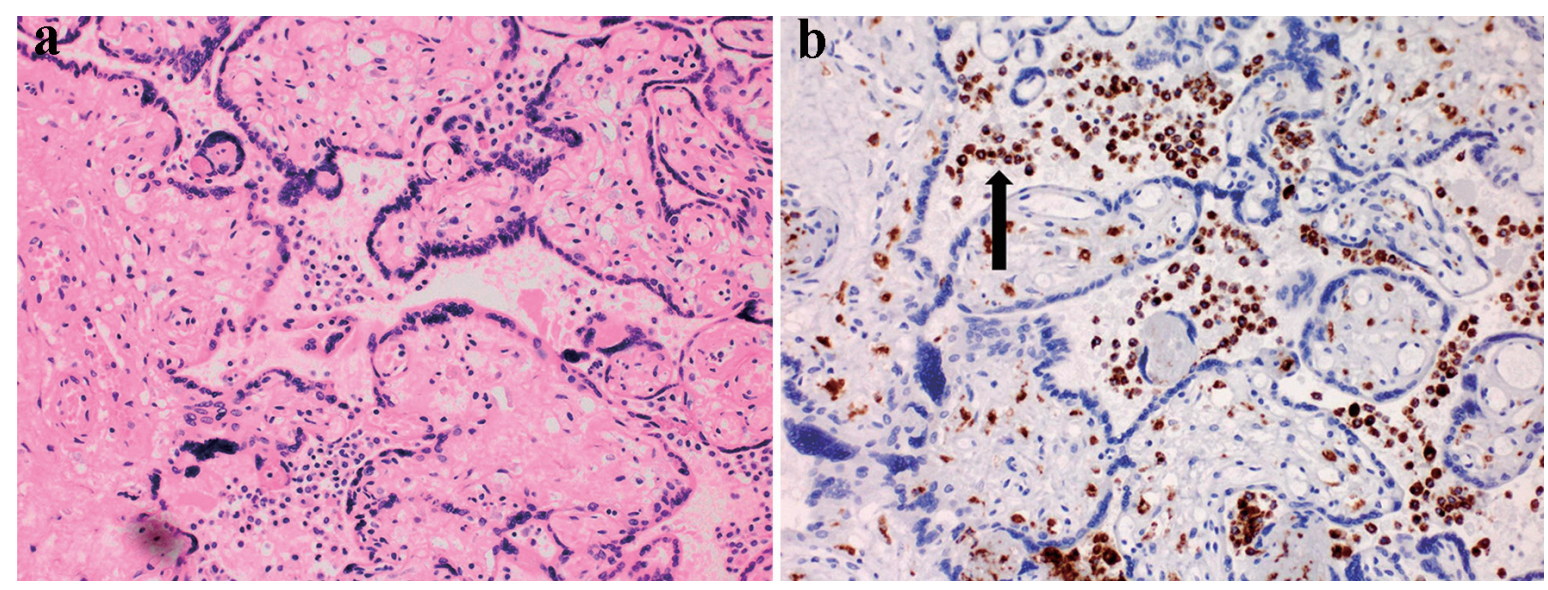

Figure 2. Placental villous histology. (a) H\&E-stained image with chorionic villi and intervillous space filled with mononuclear cells ( 20 magnification). (b) IHC image with chorionic villi and cells both in villi and in the "maternal" intervillous space (+) for CD68 (arrow), a macrophage marker. H\&E: hematoxylin and eosin; IHC: immunohistochemistry. 
ALP in pregnancy and placental dysfunction, confirmed by placental pathology.

\section{Discussion}

ALP is a ubiquitous enzyme produced in multiple tissues throughout the body. Of primary importance in our study is the placental isoenzyme. PLAP is produced by the syncytiotrophoblastic plasma membranes during the second and third trimesters of pregnancy. As gestation progresses, the concentration of PLAP rises progressively [2]. This rise may be attributed to detachment of ALP from the membrane with release into the maternal circulation. In a healthy pregnant woman, PALP rises to a level 2 - 3 times higher than that of a non-pregnant woman and remains in maternal circulation until approximately 7 days postpartum. Albeit only a few reported cases of markedly elevated ALP during pregnancy, it has been noted to be a marker for placental insufficiency, low birth weight and preterm delivery [3-9]. Similarly, elevated circulating ALP may be a marker of placental injury [10]. The elevated levels of serum PLAP and ALP in a pregnant, hypertensive woman may be attributed to placental dysfunction, resulting in an increased serum concentration of the enzyme. The mechanism of increase seen in hypertensive disorders of pregnancy is thought to be due to overactive shedding of syncytiotrophoblasts with subsequent necrosis and apo necrosis of the syncytiotrophoblast particles [11]. This damage may be seen microscopically, with placental pathology demonstrating placental infarction and damage to villous syncytiotrophoblasts.

ALP is a membrane-associated enzyme and participates in membrane transport mechanisms that may be imperative for placental metabolism. An unusually high or acutely rising ALP is a useful tool to identify high-risk pregnancies and to predict underlying placental damage. Furthermore, an increasing ratio of PALP to ALP may be used as an index of placental function, suggesting an abnormally functioning placenta. A more practical approach to assessing an abnormally rising ALP would be to utilize the heat-stable ALP isoenzyme, or PLAP, in combination with the PLAP/ALP ratio. The above tests are simple to assay, reliable, economical and sensitive. Hypertensive disorders of pregnancy (HDPs) continue to be a major cause of maternal and fetal morbidity and mortality in developing countries. The prevention of morbidity associated with HDP lies in early detection of biomarkers which will aid in the assessment of the severity of disease and in predicting fetomaternal prognosis.

In conclusion, a unique feature of our case is markedly elevated ALP levels with significant placental changes in the form of diffuse villous edema and chorionic plate vessels with dense embedded mural thrombus.

\section{Learning points}

Abnormally elevated and rising ALP may be a potential marker for placental dysfunction with subsequent poor perinatal outcomes.
Close monitoring of the absolute value and degree of rise in ALP may hold significant clinical importance in predicting impaired placental function and ultimately indicating earlier delivery.

\section{Acknowledgments}

None to declare.

\section{Financial Disclosure}

None to declare.

\section{Conflict of Interest}

None to declare.

\section{Informed Consent}

Informed consent has been obtained.

\section{Author Contributions}

Tracey Juron performed review of literature and worked together with Ena Arora to write up the case report. Aleksandr Fuks provided mentorship and peer review. Carolyn Salafia performed placental pathology.

\section{Data Availability}

Any inquiries regarding supporting data availability of this study should be directed to the corresponding author.

\section{References}

1. Kwo PY, Cohen SM, Lim JK. ACG clinical guideline: evaluation of abnormal liver chemistries. Am J Gastroenterol. 2017;112(1):18-35.

2. Safarova A, Bige O, Dogan E, Kaymaz C. Origin and significance of extremely elevated serum alkaline phosphatase activity during normal pregnancy. Turkiye Klinikleri J Gynecol Obst. 2007;17:405-408.

3. Moawad AH, Goldenberg RL, Mercer B, Meis PJ, Iams JD, Das A, Caritis SN, et al. The Preterm Prediction Study: the value of serum alkaline phosphatase, alpha-fetoprotein, plasma corticotropin-releasing hormone, and other serum markers for the prediction of spontaneous preterm birth. Am J Obstet Gynecol. 2002;186(5):990-996.

4. Bashiri A, Katz O, Maor E, Sheiner E, Pack I, Mazor M. Positive placental staining for alkaline phosphatase corresponding with extreme elevation of serum alkaline 
phosphatase during pregnancy. Arch Gynecol Obstet. 2007;275(3):211-214.

5. Ferianec V, Linhartova L. Extreme elevation of placental alkaline phosphatase as a marker of preterm delivery, placental insufficiency and low birth weight. Neuro Endocrinol Lett. 2011;32(2):154-157.

6. Meyer RE, Thompson SJ, Addy CL, Garrison CZ, Best RG. Maternal serum placental alkaline phosphatase level and risk for preterm delivery. Am J Obstet Gynecol. 1995;173(1):181-186.

7. Rajagambeeram R, Abu Raghavan S, Ghosh S, Basu S, Ramasamy R, Murugaiyan SB. Diagnostic utility of heat stable alkaline phosphatase in hypertensive disorders of pregnancy. J Clin Diagn Res. 2014;8(11):CC10-13.

8. Vongthavaravat V, Nurnberger MM, Balodimos N, Blan- chette H, Koff RS. Isolated elevation of serum alkaline phosphatase level in an uncomplicated pregnancy: a case report. Am J Obstet Gynecol. 2000;183(2):505-506.

9. Heazell AE, Judge JK, Bhatti NR. A case of isolated peripartum elevation of alkaline phosphatase in pregnancy complicated by gestational diabetes. J Matern Fetal Neonatal Med. 2006;19(5):311-313.

10. Ranganath L, Taylor W, John L, Alfirevic Z. Biochemical diagnosis of placental infarction/damage: acutely rising alkaline phosphatase. Ann Clin Biochem. 2008;45(Pt 3):335-338.

11. Hutchinson ES, Brownbill P, Jones NW, Abrahams VM, Baker PN, Sibley CP, Crocker IP. Utero-placental haemodynamics in the pathogenesis of pre-eclampsia. Placenta. 2009;30(7):634-641. 